\title{
Pathotyping and Genetic Characterization of Avian Avulavirus-1 and Low Pathogenicity H9N2 Avian Influenza Viruses Isolated from Punjab, Pakistan
}

\author{
Irfan Irshad ${ }^{1}$, Asim Aslam ${ }^{1, *}$, Muhammad Yasin Tipu ${ }^{1}$, Kamran Ashraf $^{2}$, \\ Beenish Zahid ${ }^{3}$ and Abdul Wajid ${ }^{4}$ \\ ${ }^{1}$ Department of Pathology, University of Veterinary and Animal Sciences, Lahore \\ ${ }^{2}$ Department of Parasitology, University of Veterinary and Animal Sciences, Lahore \\ ${ }^{3}$ Department of Pathology, Khan Bahadar Choudhry Mushtaq Ahmed, College of \\ Veterinary and Animal Sciences, Narowal \\ ${ }^{4}$ Department of Biotechnology, Virtual University of Pakistan
}

\begin{abstract}
A B S T RA C T
Virulent strains of AAvV-1s and low-pathogenic H9N2 avian influenza viruses are presently endemic in Pakistan and repeated outbreaks are continuously being reported with high mortality in poultry and nonpoultry avian species. In this study, five AAvV-1s have been pathotyped and genetically characterized from vaccinated birds collected between 2013-14. A phylogenetic analysis revealed that all the isolates belonged to sub-genotype VIIi with high similarity of 97.9 to $99.8 \%$ with similar viruses in this clade. Analysis of the hemagglutinin (HA) gene sequences of the two AIV was performed and phylogenetic analysis reveals genetically closely-related to H9N2 viruses classified into Mideast group-B and sublineage B2. The two strains classified as LPAIV in poultry based on amino acid sequence at the proteolytic cleavage-site of the HA gene with PAKSSR/G. Our findings highlight the potential risk of ND and AI in poultry and continued active surveillance is needed to monitor the transmission of these viruses.
\end{abstract}
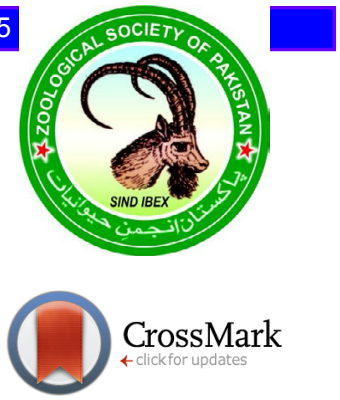

\section{INTRODUCTION}

$\mathrm{P}$ oultry farming industry is the second biggest industry of Pakistan and is always overwhelmed by multiple avian pathogens. Repeated outbreaks of ND and AI have been reported from the last three decades and are of great economic concern for poultry production facilities in Pakistan. AIV subtype H9N2 and ND are two of the most significant diseases of poultry caused by type A orthomyxoviruses and Avian Avulavirus-1 (Previously referred to as APMV-1 or Newcastle disease virus). ND is caused by virulent strains of Avian Avulavirus-1 (AAvV1) is affecting domestic and wild bird species (Wajid et al., 2017). AAvV-1 is classified as a member of family Paramyxoviridae of the genus Avulavirus and contains a negative sense, single stranded, non-segmented RNA genome of approx. $15.2 \mathrm{~kb}$ and encoded six structural proteins 3-N-P-M-F-HN-L-5. Genetically, two classes of $\mathrm{AAvV}-1$ based on the phylogenetic analysis of complete F-gene sequences. Class-1 contains viruses are mostly avirulent with a single genotype and Class-II contains eighteen genotypes (I-XVIII) together with several subgenotypes are present in both wild-birds and poultry

\footnotetext{
Corresponding author: drasimaslamch@uvas.edu.pk 0030-9923/2020/0001-0001 \$ 9.00/0

Copyright 2020 Zoological Society of Pakistan
}

(Diel et al., 2012). To date, several genotype and subgenotypes of AAvV-1 have been reported in Pakistan (VIId, VIIf, XIIIb, VI, II) and newly identified sub-genotype VIIi $\mathrm{AAvV}-1$ is highly pathogenic causing high mortality in vaccinated poultry (Rehmani et al., 2015).

AIVs are encoded by eight segments of singlestranded, enveloped and negative-sense RNA genome of $13.5 \mathrm{~kb}$. Since the first outbreak of H9N2 reported in 1998, Pakistan poultry industry has been experienced significant economic losses due to LPAIV (Lee et al., 2016). The H9N2 viruses have become prevalent in poultry in Pakistan and have evolved through re-assortment with highly pathogenic avian influenza viruses $\mathrm{H} 5 \mathrm{~N} 1$ and $\mathrm{H} 7 \mathrm{~N} 3$, generating a novel genotypes of H9N2 (Munir et al., 2013). The novel genotypes of H9N2 contain mammalian host specific markers, recent surveillance is important to better understand any continuing public health risk. Here we report complete F-gene sequences of five virulent strains of AAvV-1 and two HA-gene sequences of H9N2 strains isolated during 2015-16 from various outbreaks have been genetically characterized.

\section{MATERIALS AND METHODS}

Sample collection and virus isolation

In this study, cloacal swabs were collected from the suspected cases, 100 each for ND and H9N2 from 
poultry farms located in Lahore, Gujranwala, Okara, Kasur and Sialkot districts of Punjab province, Pakistan. The swabs were suspended in phosphate buffered saline (PBS) containing antibiotic, streptomycin $(1000 \mathrm{U} / \mathrm{ml})$ and penicillin $(1000 \mathrm{U} / \mathrm{ml})$. Samples were inoculated into the allantoic cavity of 9-10 day-old embryonated chicken eggs-ECE (specific NDV-antibodies free) and (Specific AIV-antibodies free) for NDV and AIV propagation. The harvested allantoic fluids were tested using a spot hemagglutination (HA) assay. The antisera against AI (H9N2) and ND was received from the OIE recognized Lab for NDV and AI at South East Poultry Research Lab, Athens, Georgia, USA.

\section{Intracerebral pathogenicity index (ICPI) assay}

The virulence of five AAvV-1 isolates was determined by ICPI assay using day-old chicks following an established procedure (OIE, 2012).

\section{RNA Extraction and Sequencing}

The genomic RNA was extracted from HA-positive allantoic fluids through TRIzol LS-reagent (Invitrogen) according to the manufacturer's protocol. Reverse transcriptase-PCR (RT-PCR) kit was used for the synthesis of first strand complementary DNA (cDNA) using random hexamer (Themro-Scientific) according to the manufacturer's recommendations. For PCR amplification of complete coding region of F-gene of five AAvV-1s was performed using primers as previously described (Wajid et al., 2017). For the amplification of complete coding region of HA-gene of two influenza viruses was performed by newly designed primers H9HA_1_F: 5'-ATGGAAGCAATATCACTGATGATTATA-3' - - and H9HA_1_R: 5'-ATCTTACTCTCAACGTCTGTCC-3' (764 base pair [bp]). H9HA_2_F: 5'-GAACCGACACAACAACAAGCG-3 and H9HA_2 R: 5'-CTATATACAAATGTTGCATCTGCATG-3' (10 $\overline{0} \overline{0}$ bp). The amplified products were sequenced by ABI-3130$X L$ genetic analyzer (ABI, Inc., CA, USA).

\section{Phylogenetic analysis}

The obtained nucleotide sequences of complete F-gene and HA-gene were aligned using BLAST. The phylogenetic analysis based on F-gene (1662 bp) and hemagglutinin gene (1683 bp) was performed with MEGA7 (Kumar et al., 2016) and the evolutionary history was inferred by using the Maximum likelihood-method with statistical analysis based on 1000 bootstrapping replicates. To infer the phylogenetic analysis of AAvV1s $(n=71)$ Avian influenza virus $(n=64)$ sequences were retrieved from GenBank.

\section{RESULTS AND DISCUSSION}

The virulent strains of AAvV-1s and LPAI H9N2 strains are endemic in Pakistan. The continuous cocirculations of these viruses in poultry production facilities causes huge direct and indirect economic losses and pose a significant threat to global food security. Consequently, it is indispensable to evaluate the degree of genetic diversity of these viruses circulating in the field and to estimate the genetic relationship with previously characterized viruses. At present, limited molecular epidemiological data are available regarding the causes of ND and AI outbreaks in vaccinated commercial poultry farms. Field observations indicate that the influence of $\mathrm{AAvV}-1$ is more severe with simultaneous circulation of LPAI strains in countries where these viruses are endemic (Bonfante et al., 2017). In this study, we surveyed the prevalence and characterization of AAvV-1s and H9N2 LPAIVs in one hundred vaccinated commercial poultry farms located in Lahore, Gujranwala, Okara, Kasur and Sialkot districts of Punjab province, Pakistan.

Table I.- Description of samples analyzed in this study.

\begin{tabular}{|c|c|c|c|c|c|c|c|c|c|}
\hline \multirow[t]{2}{*}{ Strain } & \multirow[t]{2}{*}{ Host } & \multirow[t]{2}{*}{ Type } & \multirow[t]{2}{*}{ City } & \multirow[t]{2}{*}{ Year } & \multirow[t]{2}{*}{ ICPI } & \multirow{2}{*}{$\begin{array}{c}\text { Cleavage site } \\
\text { (F-gene aa112-117) }\end{array}$} & \multirow[t]{2}{*}{ Genotype } & \multicolumn{2}{|c|}{ HI titer } \\
\hline & & & & & & & & Mean & SD \\
\hline \multicolumn{10}{|l|}{ AAvV-1s } \\
\hline Chicken/Pak/Lahore/AW-98/2013 & Chicken & Farm & Lahore & 2013 & 1.61 & RRQKRF & VIIi & 6.9 & 1.06 \\
\hline Chicken/Pak/Gujran/AW-122/2013 & Chicken & Farm & Gujranwala & 2013 & 1.66 & RRQKRF & VIIi & 6.0 & 0.88 \\
\hline Chicken/Pak/Lahore/AW-269/2013 & Chicken & Farm & Lahore & 2013 & 1.83 & RRQKRF & VIIi & 5.8 & 2.39 \\
\hline Chicken/Pak/Sialkot/AW-101/2014 & Chicken & Farm & Sialkot & 2014 & 1.71 & RRQKRF & VIIi & 6.0 & 1.02 \\
\hline Chicken/Pak/Okara/AW-61/2014 & Chicken & Farm & Okara & 2014 & 1.70 & RRQKRF & VIIi & 6.8 & 1.59 \\
\hline H9N2 & & & & & & HA-gene & \multicolumn{3}{|c|}{ Pathogenicity } \\
\hline A/Chicken/Pak/AW03/2014(H9N2) & Chicken & Farm & Kasur & 2014 & - & $\mathrm{PAKSSR} / \mathrm{G}$ & LPAIV & 6.4 & 1.05 \\
\hline A/Chicken/Pak/RZQ25/2014(H9N2) & Chicken & Farm & Lahore & 2014 & - & PAKSSR/G & LPAIV & 6.9 & 1.06 \\
\hline
\end{tabular}


Here, we discovered the continual isolation of genetically very closely related virulent $\mathrm{AAvV}-1$ strains from poultry and wild birds species over a seven years period (Wajid et al., 2017; Miller et al., 2015; Munir et al., 2010). Sequencing of the region corresponding to fusion protein cleavage site of five studied AAvV-1 strains revealed its amino acid sequence ${ }^{112}$-RRQKRF- ${ }^{117}$ typical for virulent form (OIE, 2012) and consistent with the determined ICPI value recorded 1.61 to 1.83 (Table I). We analyzed the evolutionary distance to compared compete F-gene of the studied AAvV-1s and other previously characterized Pakistani viruses. The nucleotide distance among the analyzed viruses was found $0.2 \%$ to $2.1 \%$. The recently isolated viruses were phylogenetically characterized by complete coding region of F-gene. Based on the set criteria by Diel et al. (2012) these isolates were classified as members of the most prevalent sub-genotype VIIi (Fig. 1). We demonstrated very close phylogenetic relationship between the current viruses with previously characterized viruses isolated from different commercial and domestic and captive non-poultry birds in country.

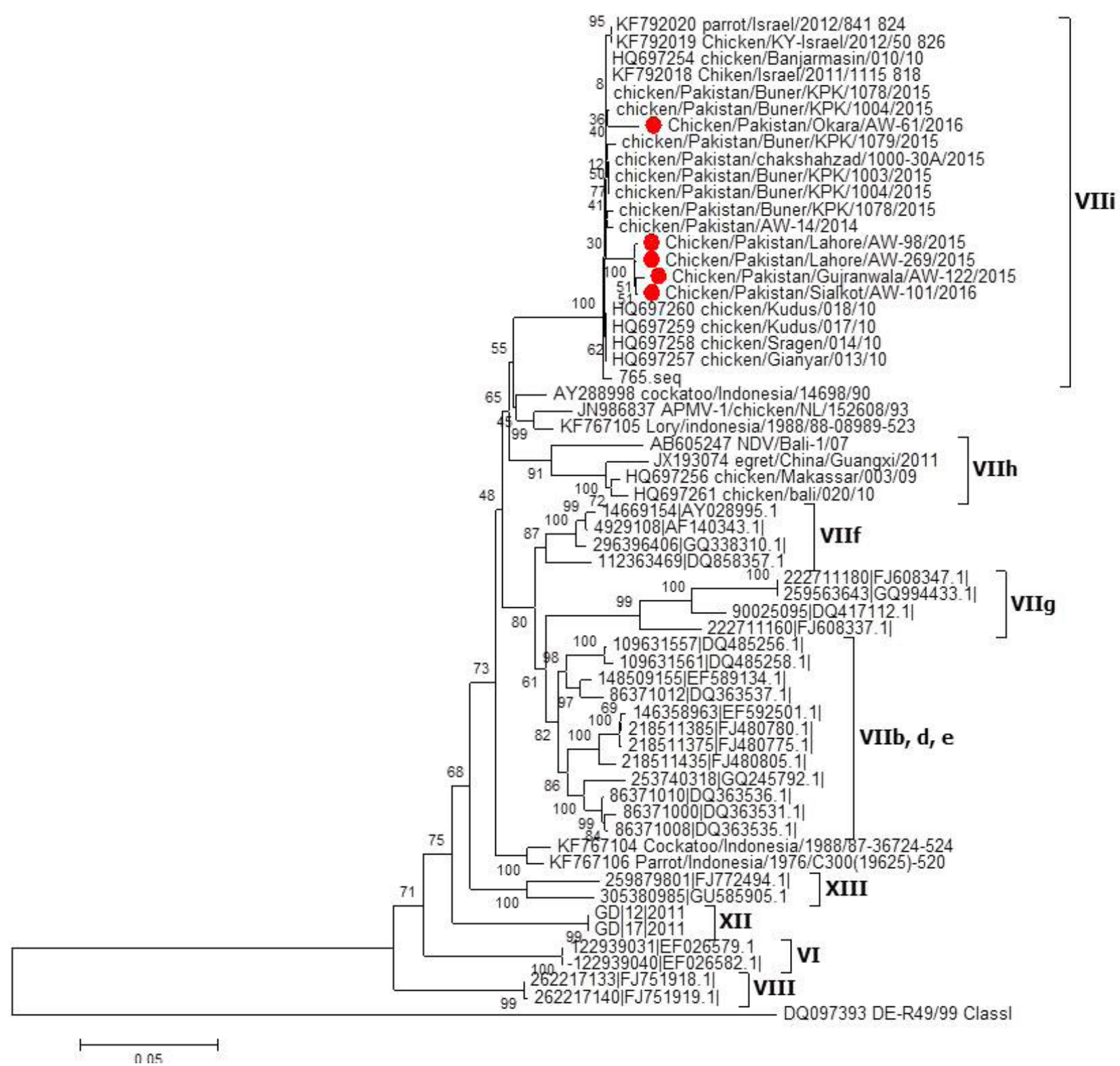

Fig. 1. Neighbour-joining analysis using the MEGA7-software of the complete nucleotide sequence of the F-gene from the five $\mathrm{AAvV}-1 \mathrm{~s}$ isolated in this study (red-filled circles). 
In order to genetically characterize two influenza viruses isolated from vaccinated commercial poultry flocks in Eastern region of Pakistan, the HA-gene was sequenced. The two Pakistani isolates tested in this study were LPAIV viruses in poultry based on aa sequence at the proteolytic cleavage site of the HA-gene PAKSSR/G. The two isolates were phylogenetically analyzed on the basis of HA-gene and compared with the representative H9N2 strains of Eurasian origin. The previous investigations demonstrated that the H9N2 strains causing diseases in poultry are divided into three lineages including Y439 or Korean-like, $\mathrm{Ch} / \mathrm{Bj} / 1 / 94$, and Qa/HK/G1/97 represented by A/duck/H-K/Y439/97, A/chicken/Beijing/1/94 and quail/ Hong-Kong/G1/97. The phylogenetic analysis reveals genetically closely related to H9N2 viruses classified into Mideast group B and clustered with other viruses belonged to India, Iran, Israel, Saudi Arabia, United Arab Emirate (UAE) and Pakistani isolates (Fig. 2). Mideast B is divided into two sub-lineages, B1 and B2, which contains viruses collected from 2004-2008 and 2008-2015, respectively. The two Pakistani H9N2 isolates collected in this study are clustered into sub-lineage B2 which contains previously characterized Pakistani viruses. The nucleotide sequence identity of Pakistani isolates demonstrated that the HAgene shared very high nucleotide sequence similarity ranged from 99-99.3\% with viruses isolated during 20152016 in sub-lineage B2.

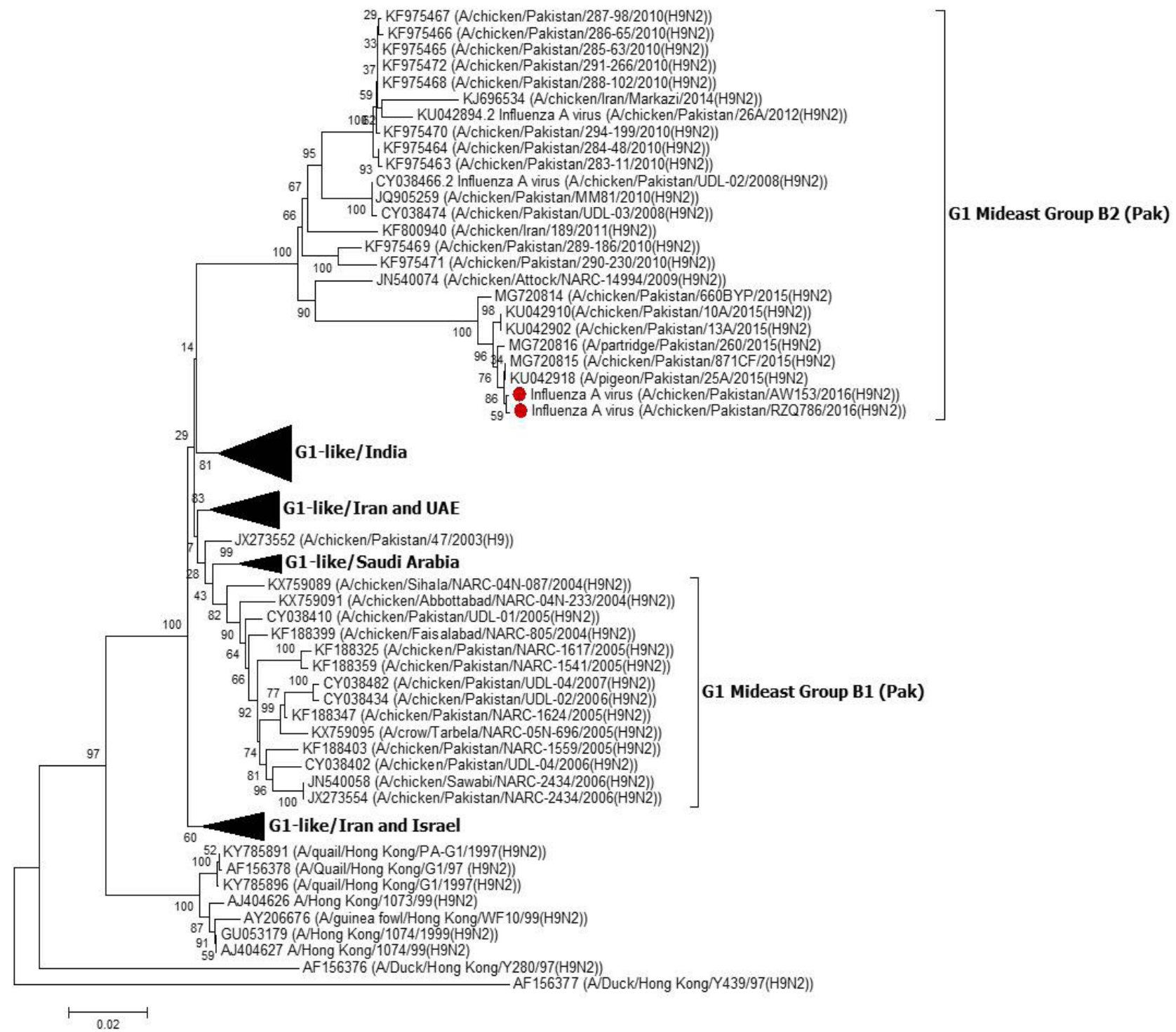

Fig. 2. Phylogenetic analysis of HA-gene sequences of two H9N2 AIVs indicated with red-filled circle. 


\section{CONCLUSION}

The present study investigated the co-circulating of virulent-AAvV-1s and LPAIVs in vaccinated poultry flocks. Although the H9N2 isolates are considered as low pathogenic, the current condition in Pakistan revealed that it could cause indeed very substantial losses in different types of poultry production. Though, there are many reasons supporting the emerging of disease mainly the migratory birds as a possible mechanism for the redistribution of virulent-AAvV-1s and LPAIVs. But other unnoticed causes may be involved such as the marketing trend i.e. the movement of infected commercial poultry, free-range rearing of backyard and captive non-poultry birds. Unfortunately, the country still lacks the international quarantine standards for interstate movement and that might be the major reason of the spread of pathogens in avian species.

\section{Statement of conflict of interest}

The authors declare no conflict of interest.

\section{REFERENCES}

Bonfante, F., Cattoli, G. and Leardini, S., 2017. Synergy or interference of H9N2 avian influenza virus with a velogenic Newcastle disease virus in chickens is dose dependent. Avian Pathol., 46: 488-496. https:// doi.org/10.1080/03079457.2017.1319904

Diel, D.G., da Silva, L.H. and Liu, H., 2012. Genetic diversity of avian paramyxovirus-type-1: proposal for a unified nomenclature and classification system of Newcastle disease virus genotypes. Infect. Genet. Evolut., 12: 1770-1779. https://doi. org/10.1016/j.meegid.2012.07.012

Kumar, S., Stecher, G. and Tamura, K., 2016. MEGA7: Molecular Evolutionary genetics analysis V7.0 for bigger datasets. Mol. Biol. Evolut., 33: 1870-1874. https://doi.org/10.1093/molbev/msw054

Lee, D.H., Swayne, D.E. and Sharma, P., 2016. H9N2 low pathogenic avian influenza in Pakistan (20122015). Vet. Rec. Open, 3: e000171. https://doi. org/10.1136/vetreco-2016-000171

Miller, P.J., Haddas, R. and Simanov, L., 2015. Identification of new sub-genotypes of virulent Newcastle disease virus with potential panzootic features. Infect. Genet. Evolut., 29: 216-229. https://doi.org/10.1016/j.meegid.2014.10.032

Munir, M., Linde, A.M. and Zohari, S., 2010. Complete genome analysis of an avian paramyxovirus type1 strain isolated in 1994 from an asymptomatic black-headed gull (Larus ridibundus) in southern Sweden. Avian Dis., 54: 923-930. https://doi. org/10.1637/9086-092409-RESNOTE.1

Munir, M., Zohari, S. and Iqbal, M., 2013. The nonstructural (NS) gene segment of H9N2 influenza virus isolated from backyard poultry in Pakistan reveals strong genetic and functional similarities to the NS-gene of highly pathogenic H5N1. Virulence, 4: 612-623. https://doi.org/10.4161/viru.26055

OIE, 2012. Manual of diagnostic tests and vaccines for terrestrial animals: mammals, birds and bees. Biological Standards Commission, World Organization for Animal Health, Paris, pp. 1-19.

Rehmani, S.F., Wajid, A. and Bibi, T., 2015 Presence of virulent Newcastle disease virus in vaccinated chickens in farms in Pakistan. J. clin. Microbiol., 53: 1715-1718. https://doi.org/10.1128/JCM.02818-14

Wajid, A., Dimitrov, K.M. and Wasim, M., 2017. Repeated isolation of virulent-Newcastle disease viruses in poultry and captive non-poultry avian species in Pakistan from 2011-2016. Prevent. Vet. Med., 142: 1-6. https://doi.org/10.1016/j. prevetmed.2017.04.010 